\title{
NorthBEAT's Capacity-to-Consent Protocol for Obtaining Informed Consent from Youth Evaluation Participants: An Alternative to Parental Consent
}

\author{
Shevaun Nadin \\ St. Joseph's Care Group \& Lakehead University \\ Mae Katt \\ Lakehead University \\ Carolyn Dewa \\ University of California, Davis \\ Chiachen Cheng
}

St. Joseph's Care Group \& Northern Ontario School of Medicine

\begin{abstract}
Ethical practice compels evaluators to obtain informed consent from evaluation participants. When those participants are minors, parental consent is routinely sought. However, seeking parental consent may not be appropriate in all evaluation contexts. This practice note presents one context (mental health services research in rural Canada) where seeking parental consent for youth' participation in research was considered unethical and unfeasible. We present a two-step "capacityto-consent" protocol that we developed to obtain consent from youth participants. This protocol offers an ethical and feasible alternative to seeking parental consent for youth. The implications for evaluation practice are discussed.
\end{abstract}

Keywords: capacity, informed consent, youth

Résumé : Pour se conformer à des pratiques éthiques, les évaluateurs doivent obtenir le consentement éclairé de participants au projet. Lorsque ces participants sont des mineurs, le consentement des parents est généralement demandé. Cependant, il n'est pas toujours possible ou souhaitable dobtenir le consentement des parents dans tous les contextes d'évaluation. La présente note de pratique présente un contexte (recherche sur les services de santé mentale en milieu rural au Canada) où l'obtention du consentement d'un parent est considérée non éthique et impossible. Nous présentons un protocole en deux étapes de "capacité à consentir » que nous avons mis au point pour obtenir le consentement de jeunes participants. Ce protocole offre une solution de rechange éthique et applicable à lobtention du consentement

Corresponding author: Shevaun Nadin, Centre for Applied Health Research, St. Joseph's Care Group, 580 N. Algoma Street, Thunder Bay, ON P7B 5G4; nadins@tbh.net 
des parents pour les jeunes. On discute des ramifications du protocole pour la pratique de l'évaluation.

Mots clés : capacité, consentement éclairé, jeunes

Ethical practice compels evaluators to obtain informed consent from evaluation participants. When those participants are minors, parental consent is routinely sought. However, some practitioners have highlighted the need to think critically about that practice, arguing that parental consent is not appropriate in all contexts. This practice note describes one such context and offers an alternative for evaluators to consider when involving youth in evaluation. The project context is described and the informed consent literature is briefly reviewed, emphasizing critiques of the parental consent requirement. The "capacity-to-consent" protocol that was used to obtain consent from youth participants (without parental consent) is then described, followed by a discussion of the protocol and its implications for evaluation practice.

\section{CONTEXT}

\section{The NorthBEAT Project}

NorthBEAT (Barriers to Early Assessment and Treatment) is a study that assessed the mental health service needs of youth ${ }^{1}$ in Northern Ontario who experience psychosis. A mixed-methods approach was adopted, which included two types of interviews with youth who had experienced psychosis: (1) semi-structured (qualitative) interviews explored participants' help-seeking and mental health service experiences, as well as their perceptions of how services could be improved; (2) structured interviews collected quantitative data to assess participants' symptoms and functional status.

Participants were recruited from across Northern Ontario, through agencies where they received services for a severe mental illness; participants were between 13 and 21 years of age. Given the age of the participants, we initially planned to seek parental consent for their participation. Upon reflection, however, the project context caused us to question the appropriateness of seeking parental consent.

\section{Concerns about seeking parental consent for youth participation in NorthBEAT}

\section{Ethical concerns}

Participants were recruited from mental health service agencies where they were accessing service for psychosis. Many youth in this region-especially Indigenous youth-do not live in the same location as their parents because they attend school outside of their communities. Some youth seek treatment while living away from their communities. Or, in some cases, their parents do not know the extent 
of their mental health issues. Requiring parental consent threatened to minimize youth's right to privacy with respect to their health information.

\section{Practical concerns}

Participants were recruited from 10 programs across Northern Ontario. This is a region with a vast geography and scarce population; many residents live in rural and remote communities. Because of the extent of the geographic area, all interviews were conducted via telephone. One concern was that requiring written parental consent before commencing interviews would hinder data collection. For example, having parents sign and return consent forms to the researcher before the interview was an unrealistic expectation because this study population is spread across rural communities where Internet and fax-machine access is unreliable. Though obtaining verbal consent (e.g., via telephone) was considered, it also posed logistical barriers. Our core concern about the practicalities of obtaining parental consent was that it would likely add to study attrition, thereby negatively affecting the generalizability and utility of the study findings.

For guidance on how to address these concerns we consulted the literature, a brief review of which is provided in the next section.

\section{ETHICAL AND METHODOLOGICAL CONSIDERATIONS}

Though requiring parental consent for youth participation in evaluation and research may seem harmless and perhaps even desirable, compelling critiques suggest that it may not be good practice (Flicker \& Guta, 2008; Heath, Charles, Crow, \& Wiles, 2007; Hester, 2004); one example was recently presented in this journal (Scott, 2013). Though an in-depth review of the literature is beyond the scope of this article, we summarize the ethical, practical, and methodological concerns that have been raised.

Ethically, parents may not know that their children are receiving services; this is especially relevant for sensitive topics such as sexual or mental health. Requiring parental consent can threaten a youth's right to privacy regarding the services they receive (Flicker \& Guta, 2008; Scott, 2013). Further, asking youth to tell their parents they are receiving services may impede the continuation of those services; for example, increased parental monitoring may dissuade or prevent youth from using services (Flicker \& Guta, 2008; Jones, Purcell, Singh, \& Finer, 2005; Reddy, Fleming, \& Swain, 2002).

Requiring parental consent may create a barrier for youth to participate in studies that could benefit them, thereby violating the ethical principle of justice (Centre of Genomics and Policy [CGP], 2012; Flicker \& Guta, 2008; Scott, 2013). For example, evaluators may be reluctant to include youth in their studies due to practical concerns about getting parental consent and legal concerns about not getting such consent (Bruzzese \& Fisher, 2003; Fisher \& Wallace, 2000; Heath et al., 2007; Hester, 2004; Rew, Taylor-Seehafer, \& Thomas, 2000). Especially for sensitive topics, youth may be unwilling to seek parental permission, or parents 
may be unwilling to give it (Flicker \& Guta, 2008; Hester, 2004; Mustanski, 2011). When youth are excluded from evaluation studies, knowledge about their needs and their service access is diminished (CGP, 2012; Flicker \& Guta, 2008; Scott, 2013), and the evaluation standard of utility is threatened (Ferris, 2000).

Practically, requiring active consent for youth participation results in reduced response rates (Courser, Shamblen, Lavrakas, Collins, \& Ditterline, 2009; Pokorny, Jason, Schoeny, Townsend, \& Curie, 2001; Shaw, Cross, Thomas, \& Zubrick, 2015; White, Hill, \& Effendi, 2004). Although reminders and incentives can increase response rates, most evaluations do not have the resources required to do so (Johnson et al., 1999; Leakey, Lunde, Koga, \& Glanz, 2004). Methodologically, requiring parental consent can affect the generalizability of evaluation findings. Requiring parental consent can create bias by under-representing the very youth whose needs we are trying to understand (Bergstrom et al., 2009; Courser et al. 2009; Doumas, Esp, \& Hausheer, 2015; Flicker \& Guta, 2008; Scott, 2013; Shaw et al., 2015; Spence, White, Adamson \& Matthews, 2015).

\section{ETHICAL GUIDELINES AND LEGAL CONSIDERATIONS}

In Canada, the Tri-Council Policy Statement: Ethical Conduct for Research Involving Humans (TCPS2) governs the ethical conduct of research with human participants (Canadian Institutes of Health Research [CIHR], Natural Sciences and Engineering Research Council of Canada, \& Social Sciences and Humanities Research Council of Canada, 2014). ${ }^{2}$ The policy offers guidelines on obtaining consent and on who is capable of providing that consent. The TCPS2 recognizes that children may lack the capacity to consent to research participation. In such cases (i.e., where children cannot decide for themselves), consent must be sought from an authorized third party (e.g., a parent) on the child's behalf. The researcher must also ascertain the child's wishes regarding their participation (i.e., obtain assent) (CIHR et al., 2014). While some have interpreted this to mean that youth are incapable of providing consent and that parental consent is required for their participation in research and evaluation (Gibson, Stasiulis, Gutfreund, McDonald, \& Dade, 2011; Scott, 2013), the TCPS2 adopts a capacity approach to consent and does not specify an age of consent (see Box 1).

The capacity approach to consent is consistent with the developmental literature which suggests that age is not a good proxy for developmental capacity (Alderson, 2007; Baylis, Downie, Kenny, \& Baylis, 1999; Miller, Drotar, \& Kodish, 2004; Schachter, Kleinman, \& Harvey, 2005). Even very young children (e.g., seven years of age) can be capable of understanding study information (Miller et al., 2004), and many developmental researchers agree that around the age of 14 , youth have the ability to provide informed consent (Flicker \& Guta, 2008; Hester, 2004; Schachter et al., 2005; Scott, 2013).

Provincial laws vary regarding when a person is considered legally competent to provide consent, and these may differentiate between consent to treatment and 


\section{Box 1. Consent, Age of Consent, and Capacity as Defined in the Tri-Council Policy Statement}

\section{Consent}

"An informed choice that is based on as complete an understanding as is reasonably possible of the purpose of the research, what it entails, and its foreseeable risks and potential benefits both to participants and others" (p. 7).

Age of Consent (a decision-making capacity approach)

"Rather than an age-based approach to consent, the TCPS2 advocates an approach based on decision-making capacity as long as it does not conflict with any laws governing research participation" (p. 32).

\section{Decision-Making Capacity}

"Refers to the ability of prospective or actual participants to understand relevant information presented about a research project, and to appreciate the potential consequences of their decision to participate or not participate" ( p. 42).

"Assessing decision-making capacity is a question of determining, at a particular point in time, whether a participant (or prospective participant) sufficiently understands the nature of a particular research project, and the risks, consequences and potential benefits associated with it" (p. 42).

Source: CIHR et al. (2014)

consent to research (Bravo et al., 2005; CGP, 2012; Schachter et al., 2005; Scott, 2013; Wildeman et al., 2012). In Ontario (NorthBEAT's context), there is no legal age of consent and no research consent-specific legislation; therefore, the onus was on the researchers to assess whether participants had the capacity to consent to participate in NorthBEAT (CGP, 2012; CIHR et al., 2014; Gibson et al., 2011; Ontario, 1996; Hiltz \& Szigeti, 2006; McDonald, 2002).

\section{Assessing capacity to consent to research participation}

A person has the capacity to consent to participate in research if she or he is able to understand information about the study and appreciate the consequences of the decision to participate (CIHR et al., 2014; Ontario, 1996) (see also Box 1). Though some instruments are available to evaluate participants' capacity to provide informed consent, they are geared to adult populations or clinical trial research and were therefore not applicable to the NorthBEAT context (Casarett, Karlawish, \& Hirschman, 2003; Hein et al., 2012; Koelch, Prestel, Singer, Schulze, \& Fegert, 2010; Moser et al., 2002; Wirshing, Wirshing, Marder, Liberman, \& Mintz, 1998; Zayas, Cabassa, \& Perez, 2005). Therefore, we developed a two-step protocol to determine whether NorthBEAT participants were capable of consenting, anticipating that there would be children under 18 years of age capable of consenting. 


\section{NORTHBEAT'S CAPACITY-TO-CONSENT PROTOCOL}

The protocol was approved by six institutional REBs and a delegate at five organizations that did not have REBs. ${ }^{3}$ The protocol was employed with all participants, regardless of their age. The protocol was a two-step process where capacity was determined by mental health clinicians (Step 1) and the researchers (Step 2); each step is elaborated below.

\section{Step 1: Inform only "capable" youth of the project}

Front-line mental health clinicians were responsible for informing potential participants about the project. Clinicians were instructed to inform only "capable" youth about the project, and to consider a youth capable if she or he could understand the study purpose and procedures-akin to being capable of consenting to treatment. To safeguard against clinician selection bias in the recruitment procedure, clinicians were instructed to inform all youth clients whom they considered capable of consenting to participate in the project.

To safeguard against coercion, the clinicians were instructed to be mindful of potential power imbalances between themselves (as service providers) and the youth (service users). The clinicians were instructed not to be coercive; their role was simply to inform youth of the project. Interested youth then contacted the researchers to learn more about the project and schedule an interview.

Detailed letters of information (LOI) were sent (via post or electronic mail) directly to participants, and they were asked to review the project details before the scheduled interview time.

\section{Step 2: Assess capacity during consent process}

At the scheduled interview time, the interviewer began the call by confirming that the youth reviewed the LOI (if it had not yet been received, the LOI was resent and the interview rescheduled). The interviewer proceeded with a consent script that mirrored the LOI. The consent script was written to be youth-friendly and at the 7th Grade level (as indicated by the Flesch-Kincaid Grade level test in Microsoft Word).

To allow the interviewer to assess capacity at the time of the interview, "capacity check" questions were embedded in the consent script (see Appendix). In line with the TCPS2 definitions (Box 1), the "capacity check" questions allowed for an assessment of whether prospective participants understood the purpose and procedures of the study and the consequences of their decision to participate. Youth who could explain the nature of the study and the risks, benefits, and consequences of participating (or not participating) were determined to be "capable" (see Appendix).

Only "capable" youth were asked to consent (see Appendix). If a participant was deemed incapable, an interview time was scheduled at which time capacity was re-assessed. For accountability purposes (and with participants' permission), this assessment was audio-recorded. 
Step 2 was employed with 44 youth aged $13-21$ years, $57 \%$ of whom were younger than 18 years. One youth (aged 17 years) was deemed to be incapable of consenting at the time of the scheduled interview. That interview was rescheduled; however, subsequent attempts to reach that youth were unsuccessful.

\section{DISCUSSION}

Informed by the "right-to-consent" and "capacity-to-consent" literatures (e.g., Flicker \& Guta, 2008; Hein et al., 2012; Mårtenson \& Fägerskiöld, 2008; Miller et al., 2004; Scott, 2013; Zayas et al., 2005), our NorthBEAT project protocol offered an ethical and feasible alternative to parental consent. We now reflect on the factors that contributed to the protocol's success, some potential limitations, and implications for evaluation practice.

One important consideration is the jurisdictional practices that determine when youth are competent to consent to participate in research. It is important to be familiar with jurisdictional legal practices and ethical guidelines that define consent and capacity, and with what is required to obtain consent for youth participation. NorthBEAT was situated in a context where parental consent was not a mandatory precursor to youth participation in research (as stipulated by neither ethical guidelines nor law). Evaluators may find CGP (2012) and Simpson (2003) helpful in this regard. We also found it helpful to work with our institutional REB representative, who gave feedback on protocol drafts before submitting for formal REB review.

A second factor contributing to the success of this protocol was the "buy-in" from the front-line clinicians who were responsible for the first step. It is also important to note that these clinicians were trained health-care professionals for whom assessing capacity for treatment is part of their standard practice; thus, they were able to make accurate capacity assessments.

We also stress the importance of the two-step procedure. It was conceivable that a youth could decompensate to becoming incapable of providing consent between Step 1 and Step 2. In one case, this did occur. While the interviewer was going through the script, it became apparent that the youth did not have the required decision-making capacity, and the interview was rescheduled. Including this second step allowed us to be certain that the youth had decision-making capacity at the time of the interview.

Another consideration for the protocol's success is that participation in NorthBEAT was "low-risk" and finite. Through single-time interviews, participants shared their experiences of help-seeking and mental health services and self-reported their current functioning and symptom severity. It is recognized that capacity varies by context, that youth may have the capacity to consent to some studies but not others, and that capacity can change over time (Alderson, 2007; Baylis et al., 1999; CIHR et al., 2014; Gibson et al., 2011; Simpson, 2003). We believe our protocol could be appropriate for evaluation contexts similar to NorthBEAT, for example, in the evaluation of sexual health programs or in other 
sensitive service areas that youth may not want to share with their parents. However, it is not clear if the protocol would be appropriate for evaluations that employ a longitudinal design or involve high-risk participation.

Another consideration is that participants were stable in their illness and residence. A key component of the protocol was our detailed LOI that they were asked to read before the scheduled call. This protocol may not be successful with transient youth. While our protocol helped address some of the barriers regarding rural/remote geography, the remoteness of some of the communities impacted the protocol's effectiveness. It was common for the youth living in remote communities to not have received the LOI by the time of the scheduled call (e.g., mail delivery to remote regions can take several days). In those instances, calls were rescheduled; in some cases, several attempts to reconnect with the youth were unsuccessful. Thus, while the protocol did address the attrition concerns raised in the introduction of this note, it was still associated with some attrition in the remote project sites.

Logistically, due to the vast amount of information to be reviewed and some of the technical language involved, there was initial concern that the script was awkward and could be off-putting to participants. But this concern seemed to be unfounded, as participants indicated that they found our consent process acceptable; as clients of mental health services, they were familiar with comprehensive consent processes.

To be clear, we are not suggesting that parental consent is never necessary. Seeking parental consent is desirable in many contexts (Gibson et al., 2011; Heath et al., 2007). There can be the added benefit of parents helping children understand the evaluation, and parental involvement recognizes the importance of family decision-making (Gibson et al., 2011). Our intent is to highlight that evaluators should consider their specific evaluation contexts and the practical, methodological, and ethical implications of requiring parental consent.

A final consideration is that evaluators have the responsibility of ensuring there is no coercion of these vulnerable youth when parents are not included in the consent process (Boman, Bohlin, Eklöf, Forsander, \& Törner, 2015; Flicker \& Guta, 2008). In NorthBEAT, various safeguards were in place to protect youth from undue influence. First, as recommended in the literature, we adopted a community-based research (CBR) approach and used youth-friendly protocols (Flicker \& Guta). The project team comprised researchers, service providers, community members, and a youth with lived experience, and the project was driven by a goal of change (mental health service improvement)-key elements of CBR. Members of the project team were very experienced in working collaboratively with youth, and the consent script was written to be youth-friendly (while adhering to mandatory REB language). Also, in Step 1, the clinicians were instructed to be mindful of power differentials and not to act coercively. It is important to note that the clinicians' role was simply to inform youth of the project; they were not involved in the consent process. Consent was obtained during Step 2 by the interviewer (someone not involved in the youths' clinical care). Voluntary 
participation was emphasized during the consent process, and the interviewer checked to ensure that the youth understood that their participation was voluntary and not linked to their mental health services (see Appendix). Lastly, the youth were invited to include someone they trusted to be part of the consent process (see Appendix). Notably, none of the participants chose this option.

\section{CONCLUSION}

We have highlighted that, in Canada, parental consent may not be legally or ethically required for youth participation in evaluation studies (CGP, 2012; CIHR et al., 2014). When evaluations involve youth participants, evaluators are compelled to critically examine the conventional practice of mandating parental consent for its ethical and legal applicability to their specific evaluation contexts. For sensitive evaluation topics, evaluators may opt for alternative procedures for obtaining informed consent from youth. NorthBEAT's capacity-to-consent protocol allowed us to maintain youth's right to privacy regarding their mental health information while addressing logistical barriers posed by seeking parental consent across a vast, rural, and remote geography. Our literature review and protocol can be helpful to evaluators facing similar dilemmas about requiring parental consent for youth participation in evaluation studies.

\section{NOTES}

1 We use the term "youth" to refer to individuals who are under the age of 18 . The term "parent" refers to parents and/or guardians (i.e., someone who is responsible for or could be the substitute decision-maker for the youth).

2 Technically the TCPS2 governs "research" and identifies "program evaluation" as exempt from REB review (CIHR et al., 2014, Chapter 2). It is noted however, that much of what evaluators do is research. Indeed, the TCPS2 definition of "research" ("an undertaking intended to extend knowledge through a disciplined inquiry and/or systematic investigation," p. 13), is consistent with the Canadian Evaluation Society's definition of evaluation ("the systematic assessment of the design, implementation, or results of an initiative for the purposes of learning or decision-making," CES, 2015). Moreover, increasingly evaluators are required to undergo REB review by their institutions or the institutions of the programs they are evaluating. Finally, even in cases where REB review is not institutionally required, the evaluation standard of propriety compels evaluators to operate according to the ethical guidelines in our jurisdictions (in Canada, the TCPS2).

3 The protocol was approved by the REBs at the researchers' institutions: St. Joseph's Care Group (SJCG), Lakehead University, and the Centre for Addictions and Mental Health. It was also approved by the REBs at three of the ten project sites that had formalized REBsHealth Sciences North, North Bay Regional Health Centre, Sault Area Hospital,- and a delegate (e.g., program manager) at five project sites that did not have formalized REBs.

\section{ACKNOWLEDGEMENTS}

This research was supported by The Sick Kids Foundation, in partnership with The Canadian Institutes of Health Research. 


\section{REFERENCES}

Alderson, P. (2007). Competent children? Minors' consent to health care treatment and research. Social Science \& Medicine, 65(11), 2272-2283. https://doi.org/10.1016/j. socscimed.2007.08.005

Baylis, F., Downie, J., Kenny, N., \& Baylis, F. (1999). Children and decision making in health research. IRB: Ethics \& Human Research, 21(4), 5-10. https://doi.org/10.2307/3564418

Bergstrom, J.P., Partington, S., Murphy, M.K., Galvao, L., Fayram, E., \& Cisler, R.A. (2009). Active consent in urban elementary schools: An examination of demographic differences in consent rates. Evaluation Review, 33(5), 481-496. https://doi. org/10.1177/0193841X09339987

Boman, Å., Bohlin, M., Eklöf, M., Forsander, G., \& Törner, M. (2015). Conceptions of diabetes and diabetes care in young people with minority backgrounds. Qualitative Health Research, 25(1), 5-15. https://doi.org/10.1177/1049732314549358

Bravo, G., Gagnon, M., Wildeman, S., Marshall, D.T., Pâquet, M., \& Dubois, M.F. (2005). Comparison of provincial and territorial legislation governing substitute consent for research. Canadian Journal on Aging, 24(3), 237-250. https://doi.org/10.1353/ cja.2005.0074

Bruzzese, J.-M., \& Fisher, C.B. (2003). Assessing and enhancing the research consent capacity of children and youth. Applied Developmental Science, 7(1), 13-26. https://doi. org/10.1207/S1532480XADS0701_2

Canadian Evaluation Society (CES). (2015). What is evaluation? Retrieved from http:// evaluationcanada.ca/what-is-evaluation

Canadian Institutes of Health Research (CIHR), Natural Sciences and Engineering Research Council of Canada, \& Social Sciences and Humanities Research Council of Canada. (2014, December). Tri-Council Policy Statement: Ethical conduct for research involving humans.

Casarett, D.J., Karlawish, J.H.T., \& Hirschman, K.B. (2003). Identifying ambulatory cancer patients at risk of impaired capacity to consent to research. Journal of Pain and Symptom Management, 26(1), 615-624. https://doi.org/10.1016/S0885-3924(03)00221-5

Centre of Genomics and Policy (CGP), Maternal Infant Child and Youth Research Network (MICYRN). (2012). Best practices for health research involving children and adolescents. Retrieved from http://www.genomicsandpolicy.org/en/best-practices-2012

Courser, M.W., Shamblen, S.R., Lavrakas, P.J., Collins, D., \& Ditterline, P. (2009). The impact of active consent procedures on nonresponse and nonresponse error in youth survey data: Evidence from a new experiment. Evaluation Review, 33(4), 370-395. https://doi.org/10.1177/0193841X09337228

Doumas, D.M., Esp, S., \& Hausheer, R. (2015). Parental consent procedures: Impact on response rates and nonresponse bias. Journal of Substance Abuse and Alcoholism, 3(2), 1031-1034.

Ferris, L.E. (2000). Legal and ethical issues in evaluating abortion services. American Journal of Evaluation, 21(3), 329-340. https://doi.org/10.1177/109821400002100304

Fisher, C.B., \& Wallace, S.A. (2000). Through the community looking glass: Reevaluating the ethical and policy implications of research on adolescent risk and psychopathology. Ethics \& Behavior, 10(2), 99-118. https://doi.org/10.1207/S15327019EB1002_01 
Flicker, S., \& Guta, A. (2008). Ethical approaches to adolescent participation in sexual health research. Journal of Adolescent Health, 42(1), 3-10. https://doi.org/10.1016/j. jadohealth.2007.07.017

Gibson, B.E., Stasiulis, E., Gutfreund, S., McDonald, M., \& Dade, L. (2011). Assessment of children's capacity to consent for research: A descriptive qualitative study of researchers' practices. Journal of Medical Ethics, 37(8), 504-509. https://doi.org/10.1136/ jme.2010.040097

Heath, S., Charles, V., Crow, G., \& Wiles, R. (2007). Informed consent, gatekeepers and go-betweens: Negotiating consent in child and youth-orientated institutions. British Educational Research Journal, 33(3), 403-417. https://doi.org/10.1080/ 01411920701243651

Hein, I.M., Troost, P.W., Lindeboom, R., de Vries, M.C., Zwaan, C.M., \& Lindauer, R.J.L. (2012). Assessing children's competence to consent in research by a standardized tool: A validity study. BMC Pediatrics, 12(1), 156-163. https://doi.org/10.1186/14712431-12-156

Hester, C.J. (2004). Adolescent consent: Choosing the right path. Issues in Comprehensive Pediatric Nursing, 27(1), 27-37. https://doi.org/10.1080/01460860490279536

Hiltz, D., \& Szigeti, A.A. (2006). A guide to consent and capacity law in Ontario. Markham, ON: LexisNexis Butterworths.

Johnson, K., Bryant, D., Rockwell, E., Moore, M., Waters Straub, B., Cummings, P., \& Wilson, C. (1999). Obtaining active parental consent for evaluation research: A case study. American Journal of Evaluation, 20(2), 239-249. https://doi. org/10.1177/109821409902000206

Jones, R.K., Purcell, A., Singh, S., \& Finer, L.B. (2005). Adolescents' reports of parental knowledge of adolescents' use of sexual health services and their reactions to mandated parental notification for prescription contraception. Journal of the American Medical Association, 293(3), 340-348. https://doi.org/10.1001/jama.293.3.340

Koelch, M., Prestel, A., Singer, H., Schulze, U., \& Fegert, J.M. (2010). Report of an initial pilot study on the feasibility of using the MacArthur Competence Assessment tool for clinical research in children and adolescents with attention-deficit/hyperactivity disorder. Journal of Child and Adolescent Psychopharmacology, 20(1), 63-67. https:// doi.org/10.1089/cap.2008.0114

Leakey, T., Lunde, K.B., Koga, K., \& Glanz, K. (2004). Written parental consent and the use of incentives in a youth smoking prevention trial: A case study from Project SPLASH. American Journal of Evaluation, 25(4), 509-523. https://doi. org/10.1177/109821400402500407

Mårtenson, E.K., \& Fägerskiöld, A.M. (2008). A review of children’s decision-making competence in health care. Journal of Clinical Nursing, 17(23), 3131-3141. https://doi. org/10.1111/j.1365-2702.2006.01920.x

McDonald, M.Z. (2002). No age of consent in Ontario. Retrieved from http://mlst.mindzplay.ws/mlst/doc/AgeOfConsent.pdf

Miller, V.A., Drotar, D., \& Kodish, E. (2004). Children's competence for assent and consent: A review of empirical findings. Ethics \& Behavior, 14(3), 255-295. https://doi. org/10.1207/s15327019eb1403_3 
Moser, D.J., Schultz, S.K., Arndt, S., Benjamin, M.L., Fleming, F.W., Brems, C.S., Paulsen, J.S., Appelbaum, P.S., \& Andreasen, N.C. (2002). Capacity to provide informed consent for participation in schizophrenia and HIV research. American Journal of Psychiatry, 159(7), 1201-1207. https://doi.org/10.1176/appi.ajp.159.7.1201

Mustanski, B. (2011). Ethical and regulatory issues with conducting sexuality research with LGBT adolescents: A call to action for a scientifically informed approach. Archives of Sexual Behavior, 40(4), 673-686. https://doi.org/10.1007/s10508-011-9745-1

Ontario. (1996). Health Care Consent Act. S.O. 1996, Chapter 2. Retrieved from https:// www.ontario.ca/laws/statute/96h02

Pokorny, S.B., Jason, L.A., Schoeny, M.E., Townsend, S.M., \& Curie, C.J. (2001). Do participation rates change when active consent procedures replace passive consent? Evaluation Review, 25(5), 567-580. https://doi.org/10.1177/0193841X0102500504

Reddy, D.M., Fleming, R., \& Swain, C. (2002). Effects of mandatory parental notification on adolescent girls' use of sexual health care services. Journal of the American Medical Association, 288(6), 710-714. https://doi.org/10.1001/jama.288.6.710

Rew, L., Taylor-Seehafer, M., \& Thomas, N. (2000). Without parental consent: Conducting research with homeless adolescents. Journal of the Society of Pediatric Nurses, 5(3), 131-138. https://doi.org/10.1111/j.1744-6155.2000.tb00098.x

Schachter, D., Kleinman, I., \& Harvey, W. (2005). Informed consent and adolescents. Canadian Journal of Psychiatry, 50(9), 534-540. https://doi.org/10.1177/070674370505000906

Scott, S. (2013). Informed consent of 16-18-year-old participants in evaluations. Canadian Journal of Program Evaluation, 28(2), 65-84.

Shaw, T., Cross, D., Thomas, L.T., \& Zubrick, S.R. (2015). Bias in student survey findings from active parental consent procedures. British Educational Research Journal, 41(2), 229-243. https://doi.org/10.1002/berj.3137

Simpson, C. (2003). Children and research participation: Who makes what decision. Health Law Review, 11, 20-29.

Spence, S., White, M., Adamson, A.J., \& Matthews, J.N.S. (2015). Does the use of passive or active consent affect consent or completion rates, or dietary data quality? Repeat cross-sectional survey among school children aged 11-12 years. BMJ Open, 5(1). https://doi.org/10.1136/bmjopen-2014-006457

White, V.M., Hill, D.J., \& Effendi, Y. (2004). How does active parental consent influence the findings of drug-use surveys in schools? Evaluation Review, 28(3), 246-260. https:// doi.org/10.1177/0193841X03259549

Wildeman, S., Bravo, G., Dubios, M.F., Cohen, C., Graham, J., Painter, K., \& Bellemare, S. (2012). Substitute decision making about research: Identifying the legally authorized representative in four Canadian provinces. McGill Journal of Law and Health, 6(1), 189-273.

Wirshing, D.A., Wirshing, W.C., Marder, S.R., Liberman, R.P., \& Mintz, J. (1998). Informed consent: Assessment of comprehension. American Journal of Psychiatry, 155(11), 1508-1511. https://doi.org/10.1176/ajp.155.11.1508

Zayas, L.H., Cabassa, L.J., \& Perez, M.C. (2005). Capacity-to-consent in psychiatric research: Development and preliminary testing of a screening tool. Research on Social Work Practice, 15(6), 545-556. https://doi.org/10.1177/1049731505275867 


\section{AUTHOR INFORMATION}

Shevaun Nadin is a program evaluator and applied health researcher with an academic appointment as external adjunct professor in the Department of Health Sciences at Lakehead University (Thunder Bay). She is also a research affiliate at the Centre for Rural and Northern Health Research and the Centre for Education and Research on Aging and Health (Lakehead University). Dr. Nadin has worked on numerous applied research and evaluation projects in Northern Ontario including independent consulting projects and projects conducted through Lakehead University and St. Joseph's Care Group. In addition to the practice of evaluation, she also teaches program evaluation and conducts research on the practice of evaluation itself. She was also the coordinator of the NorthBEAT Project.

Mae Katt is a Primary Health Care Nurse Practitioner. Her nursing career has been diverse and includes primary health care, mental health and addictions, adolescent health, maternal and child health, and community health. She has a strong health policy and research background in community development, youth suicide, early psychosis, cancer care, health human resources, and acquired brain injury. She was appointed by the Ontario Minister of Health to the Mental Health and Addictions Leadership Council responsible for the implementation of Phase 2 of the Mental Health and Addictions Strategy. She has also worked as senior administrator responsible for First Nations health programs, both as Regional Director (Ontario) for Health Canada's First Nations and Inuit Health, and as Health Director at Nishnawbe Aski Nation. She is a member of Temagami First Nation (Ojibway).

Carolyn S. Dewa is a Professor in the Department of Psychiatry at the University of California, Davis. She is currently the Director of the Department's Mental Health Services Research and Evaluation Program. She led the Centre for Addiction and Mental Health's Centre for Research on Employment and Workplace Health and was a Professor at the University of Toronto. Her awards included an Ontario Ministry of Health and Long-Term Care Career Scientist Award and a Canadian Institutes of Health Research/Public Health Agency of Canada Applied Public Health Chair to develop effective interventions for Mental Illness and Mental Health in the Working Population. She has also been a fellow at the Harvard Medical School.

Chiachen Cheng is a Child \& Adolescent, Adult Psychiatrist and Physician Researcher. Dr. Cheng completed her Child \& Adolescent and Adult Psychiatry residencies at McMaster University and a Masters of Public Health at Harvard University. She was the Medical Director of First Place Clinic and Regional Resource Centre since its inception in 2006 until 2017, and is past co-chair of the Early Psychosis Intervention Ontario Network (EPION). Dr. Cheng's research interests are in Early Psychosis Intervention (EPI) and evidence based practice as it interfaces with pediatric mental health policy. Her research has examined program implementation and development, fidelity to Ontario's EPI standards, evaluation of EPI training for health-care workers, delivery of EPI services in rural and remote regions, and pathways to care among vulnerable populations. Dr. Cheng was the lead of the NorthBEAT Project. 


\section{Appendix: Example Script for Obtaining Consent from Youth Participants}

The script is not intended as a model, but rather as an example that evaluators may find useful for informing their protocols. Evaluators are encouraged to consider their evaluation contexts in relation to the points brought up in the body of this note when considering whether to use and/or adapt this script to their particular contexts.

This example is an abbreviated version of NorthBEAT's script that is meant to give readers a sense of the flow and capacity-check information. To save space, we have omitted many of the study-specific details.

\section{INTRODUCE YOURSELF AND THE RESEARCH:}

Interviewer [I]: My name is $\{\mathrm{xxx}\}$. I am the coordinator of the NorthBEAT project. I'd like to go over that Letter of Information in some detail with you now. Okay? And, I want to let you know that I am audio recording this just so that I can document that I am explaining NorthBEAT to you. I will erase the tape if you decide not to participate. Is that okay? Everything you say will be kept confidential.

Ok thank you, and also you are welcome to invite someone to listen in on this while I explain NorthBEAT and ask if you want to take part. Do you have someone there with you that you'd like to ask to sit in? (e.g., parent, grandparent, sister/ brother).

If youth would like to invite a support person, but that person is currently unavailable, reschedule the interview for a time the support person is available. Otherwise, continue with the script.

Great, so-This project is being funded by.... The leader of the NorthBEAT project is.... Other members of the team are....

The goal of NorthBEAT is to.... You've been invited to take part because.... If you choose to take part, you would participate in an interview with me. Before I explain the interview, and what we will do with the information, do you have any questions about the project?

Participant [P]: Response.

I: If YES, answer questions. Once questions are answered, continue with the script. 


\section{${ }^{*}$ CAPACITY CHECK ${ }^{* *}$ :}

Before explaining what taking part would involve, I want to make sure you understand what NorthBEAT is about. Can you please explain what NorthBEAT is and what we are doing?

If participant is able to explain, continue with the script. If not, thank them for their time, and terminate the interview.

P: Response.

\section{EXPLAIN WHAT PARTICIPANTS WILL BE ASKED TO DO, AND HOW THEIR INFORMATION WILL BE USED:}

Okay good. Now, let me explain what taking part in NorthBEAT would involve, and what will happen with the results.

If you agree to take part, I will ask you some questions about your experiences with mental health services.... I will tape the interview to make sure that we understand you correctly.... If you complete the interview, I will send you an honorarium $\}$. Your answers will help us understand.... The information we learn through NorthBEAT will be shared with....

Do you have any questions?

P: Response.

I: If YES, answer questions, or explain that I or RA will get back to them with the answer.

Once questions are answered, continue with the script.

\section{${ }^{* *}$ CAPACITY CHECK**:}

Okay great. I want to make sure that you understand what I am asking you to do. Can you please describe to me what I have asked you to do?

If participant is able to explain, continue with the script. If not, thank them for their time, and terminate the interview.

P: Response.

\section{DESCRIBE THE CONFIDENTIALITY PROCEDURES:}

I: I would now like to go over some information about how I will protect your privacy.

The project assistant and I know who you are, as that was necessary to contact you. What you tell me will not be connected to your name or contact information. 
Your answers will be combined with the answers of other people.... No-one will be named in any of the reports.... Your answers (with your name removed) will be stored at....

We might contact your mental health worker in case of emergency ... but we will not tell them how you answered any of the questions.... \{Mandatory REB statements about who can access the research records\}....

Do you have any questions about your privacy in this project?

P: Response.

Once questions are answered, continue with the script.

\section{DESCRIBE POTENTIAL RISKS/BENEFITS AND COMPENSATION:}

Now let me explain some potential risks and benefits of taking part.

First let me talk about the risks....

Do you have any questions about the risks of the project?

P: Response.

I: If YES, answer questions. Once questions are answered, continue with the script. In terms of benefits...

Do you have any questions about the benefits of the project?

P: Response.

I: If YES, answer questions. Once questions are answered, continue with the script.

\section{**CAPACITY CHECK**:}

I want to make sure you understand the possible risks and benefits to participating. Can you please explain the risks?

If participant is able to explain, continue with the script. If not, thank them for their time, and terminate the interview.

P: Response.

And the benefits?

P: Response.

Okay, just a few more things. I want to explain that your participation is voluntary. 


\section{EMPHASIZE VOLUNTARY PARTICIPATION AND PARTICIPANTS' RIGHTS:}

It is completely up to you whether you participate; you can say yes or no. Taking part in NorthBEAT will not affect your current care...

Do you have any questions?

P: Response.

I: If YES, answer questions. Once questions are answered, continue with the script.

\section{GIVE PARTICIPANT CHANCE TO ABSORB THE INFORMATION, AND ASK ANY QUESTIONS ABOUT THE PROJECT:}

Do you have any other questions at all about the study and what I am asking you to do?

P: Response.

I: If YES, answer questions. Once questions are answered, continue with the script. $[\ldots]$

Next I am going to ask you for your permission to participate. Do you feel you have been given enough information to decide whether you'd like to take part in NorthBEAT?

P: Response.

I: If YES, continue. If NO, probe. E.g., What else would you like to know? What are your concerns? Once questions are answered, continue with the script.

\section{${ }^{* *}$ RE-ASSESS CAPACITY**:}

Now I would like to ask for your formal consent to participate in NorthBEAT. But first, I'll ask: do you understand what you are being asked to agree to?

If participant is able to explain, continue with the script. If not, thank them for their time, and terminate the interview.

P: Response.

I: If YES, continue. If NO, probe. Once questions are answered, continue with the script.

Do you understand the consequences of agreeing to participate?

P: Response. 
I:If YES, continue. If NO, probe. Do you understand the consequences of deciding not to participate?

${ }^{*}$ Once questions are answered, continue with the script AND record capacity assessment complete and answers on the INFORMED CONSENT sheet.

\section{OBTAIN CONSENT}

Okay, now I will ask for your permission. Do you agree that I have described the study information sheet for the NorthBEAT project to you, and that you have a copy of that sheet?

P: Response.

I: If yes, continue. If no, explain further and repeat.

Do you agree that you have had the opportunity to consider the information and ask questions?

P: Response.

I: If yes, continue. If no, probe.

Do you understand that the purpose of NorthBEAT is to ...?

P: Response.

I: If yes, continue. If no, probe.

Do you understand that taking part in NorthBEAT is your choice and that you can choose to stop at any time, and that by taking part you do not waive any of your rights?

P: Response.

I: If yes, continue. If no, probe.

Do you understand that your answers will be kept private, and reported in combination with others?

P: Response.

I: If yes, continue. If no, probe.

Do you agree to take part in NorthBEAT by being interviewed?

P: Response.

I: If yes, continue. If no, thank them for their time and terminate the phone call.

Do you agree to have the interview audio tape recorded? 
P: Response.

I: If yes, continue. If no, thank them for their time and terminate the phone call.

Okay, Thank you for signing on! We are very interested in hearing your opinion so that we understand how to improve the mental health system. Now I will begin asking you the interview questions.

* If youth has invited a support person for the decision making process, remind the person that the decision-making portion is now complete, and the "private" interview portion will begin now. Only begin asking the interview questions once the youth is in a private setting. 